\title{
Genetic variants of MARCO are associated with susceptibility to pulmonary tuberculosis in a Gambian population
}

Dawn ME Bowdish ${ }^{1 *}$, Kaori Sakamoto ${ }^{2}$, Nathan A Lack ${ }^{3}$, Philip C Hill ${ }^{4,5}$, Giorgio Sirugo ${ }^{4,6}$, Melanie J Newport ${ }^{7}$, Siamon Gordon ${ }^{8}$, Adrian VS Hill ${ }^{9}$ and Fredrick O Vannberg ${ }^{10}$

\begin{abstract}
Background: The two major class A scavenger receptors are scavenger receptor A (SRA), which is constitutively expressed on most macrophage populations, and macrophage receptor with collagenous structure (MARCO), which is constitutively expressed on a more restricted subset of macrophages, (e.g. alveolar macrophages) but whose expression increases on most macrophages during the course of infection. Although the primary role of SRA appears to be clearance of modified host proteins and lipids, mice defective in expression of either MARCO or SRA are immunocompromised in multiple models of infection and in vitro assays, the scavenger receptors have been demonstrated to bind bacteria and to enhance pro-inflammatory signalling to many bacterial lung pathogens; however their importance in Mycobacterium tuberculosis infection, is less clear.
\end{abstract}

Methods: To determine whether polymorphisms in either SRA or MARCO were associated with tuberculosis, a case-control study of was performed. DNA samples from newly-detected, smear-positive, pulmonary tuberculosis cases were collected from The Gambia. Controls for this study consisted of DNA from cord bloods obtained from routine births at local Gambian health clinics. Informed written consent was obtained from patients or their parents or guardians. Ethical approval was provided by the joint The Gambian Government/MRC Joint Ethics Committee.

Results: We studied the frequencies of 25 polymorphisms of MSR1 (SRA) and 22 in MARCO in individuals with tuberculosis $(n=1284)$ and matched controls $(n=1349)$. No SNPs within the gene encoding or within $1 \mathrm{~kb}$ of the promoter sequence of MSR1 were associated with either susceptibility or resistance to tuberculosis. Three SNPs in MARCO (rs4491733, Mantel-Haenszel $2 \times 2 \times 2=6.5, p=0.001$, rs 12998782, Mantel-Haenszel $2 \times 2 \times 2=6.59, p=0.001$, rs13389814 Mantel-Haenszel $2 \times 2 \times 2=6.9, p=0.0009$ ) were associated with susceptibility to tuberculosis and one (rs7559955, Mantel-Haenszel $2 \times 2 \times 2=6.9, p=0.0009$ ) was associated with resistance to tuberculosis.

Conclusions: These findings identify MARCO as a potentially important receptor in the host response to tuberculosis. Keywords: Scavenger receptors, Mycobacterium tuberculosis, Single nucleotide polymorphisms, Case control study, MARCO

\footnotetext{
* Correspondence: bowdish@mcmaster.ca

${ }^{1}$ McMaster Immunology Research Centre, McMaster University, Hamilton, ON L8S 4K1, Canada

Full list of author information is available at the end of the article
} 


\section{Background}

Mycobacterium tuberculosis, the causative agent of tuberculosis, is one of few pathogens that infects and persists in host macrophages. Consequently it has successfully colonized up to a third of the world's population and of those, many millions develop active disease [1]. Phagocytosis of M. tuberculosis is mediated by a number of receptors including the mannose receptor and DC-SIGN, which recognise mannose-capped lipoarabinomannan (Man-lam) $[2,3]$, complement receptor via recognition of opsonincoated bacteria [4], and others [5]. Although the class A scavenger receptors, SRA (class A scavenger receptor) and MARCO (macrophage receptor with collagenous structure), are broadly classified as "phagocytic receptors", and have been demonstrated to internalize mycobacterial species such as M. leprae [6], M. avium [7], M. bovis Bacille Calmette-Guérin [8,9], and $M$. tuberculosis [10,11], it has been proposed that there is functional redundancy in mycobacterial uptake by macrophages so that any individual receptor is dispensable $[10,12]$. Intriguingly, whereas uptake of M. tuberculosis into alveolar macrophages during the course of acute infection may be mediated by many equivalent receptors, long-term extra-pulmonary persistence (e.g. in the adipose tissue), may be mediated through scavenger receptor uptake [13].

Although the importance of phagocytic receptors in uptake of M. tuberculosis is not entirely clear, their role in induction of pro-inflammatory responses appears to be more straightforward. Class A and B scavenger receptors are required for maximal cytokine responses to mycobacterial lipoarabinomannans [14] and lipopeptides [15], and both the class A scavenger receptors and the C-type lectin, Mincle, are required for optimal toll-like receptor (TLR) and Syk/Card9 signaling responses to mycobacterial trehalose dimycolate (cord factor), respectively $[16,17]$. The biological importance of this enhancement of cytokine responses remains to be fully elucidated; however, in lung infection models of $M$. tuberculosis, the absence of SRA is protective [18], but in models of disseminated disease, its absence is fatal $[8,11,19]$.

To some degree, the lack of clarity surrounding whether these receptors are of key importance in host defence towards tuberculosis is probably due to deficiencies in the mouse model. The lung pathology of tuberculosis is sufficiently different in mice, in that many of the hallmark features of disease (e.g. granulomas) do not occur, and genes found to be associated in human studies are not necessarily associated with murine susceptibility and vice versa (reviewed in [20]). In order to determine whether the class A scavenger receptors are crucial to protection against human disease, we have performed a case-control study of single nucleotide polymorphisms (SNPs) using samples from a well-described Gambian population [21,22]. We demonstrate that polymorphisms in MARCO, but not MSR1, the gene encoding SRA, are associated with tuberculosis infection in the Gambian population. Encouragingly, these results are consistent with a recently published case-control study in a Chinese Han population ( $\mathrm{n}=923$ cases and 1033 controls) [23]. In both the Chinese Han and Gambian populations, SNPs in intron 1, which we identify as a putative alternative promoter site, are associated with susceptibility to tuberculosis, implying that changes in MARCO function or expression contribute to host defence against tuberculosis.

\section{Methods}

\section{Patient DNA Samples}

DNA samples from newly-detected, smear-positive, pulmonary tuberculosis cases and healthy controls were collected from The Gambia as described [21,22]. Samples were comprised of cases and controls (please see Table 1 for details). Gambian pulmonary tuberculosis cases $(n=1,498)$ presented with a compatible clinical picture of tuberculosis and were diagnosed with culture or smear positivity; all cases that were smear positive but culture negative had radiographic confirmation. Exclusion criteria included presentation of autoimmune, cancer, or other diseases, such as HIV-1, which are known to impact host immunity. The majority (>95\%) of the tuberculosis cohort was screened for HIV-1, with positive cases excluded from the study because HIV infection increases the risk of tuberculosis. Gambian controls $(n=1,496)$ were recruited from routine births at local Gambian health clinics. Some samples were removed from the study due to quality control issues, including low genotype frequency and relatedness, leaving 1284 cases and 1349 controls for the final analysis. These cases and controls have been previously analyzed studying tuberculosis (46) and malaria [24], and we have a detailed understanding of the underlying ethnic stratification through genome-wide SNP data. Logistic regression based upon 6-axis of variation (to reduce the impact of

Table 1 Characteristics of study population

\begin{tabular}{llrlrrrr}
\hline & Status & Control & & Case & & Total & P value $^{*}$ \\
\hline Gender & Male & 712 & $40.6 \%$ & 1043 & $59.4 \%$ & 1755 & $<0.001$ \\
& Female & 784 & $63.3 \%$ & 455 & $36.7 \%$ & 1239 & \\
Ethnicity & Mandinka & 454 & $47.8 \%$ & 495 & $52.2 \%$ & 949 & 0.250 \\
& Jola & 287 & $56.9 \%$ & 217 & $43.1 \%$ & 504 & 0.004 \\
& Fula & 296 & $58.7 \%$ & 208 & $41.3 \%$ & 504 & $<0.001$ \\
& Wollof & 263 & $71.3 \%$ & 106 & $28.7 \%$ & 369 & $<0.001$ \\
& Other & 190 & $28.8 \%$ & 470 & $71.2 \%$ & 660 & $<0.001$ \\
& Unknown & 6 & $75.0 \%$ & 2 & $25.0 \%$ & 8 & \\
& Total & 1496 & & 1498 & & 2994 & \\
\hline
\end{tabular}

* Standard Chi-Square tests with 1 degree of freedom were performed for comparing groups. 
ethnicity on the final association analysis) did not affect our final conclusions. Informed written consent was obtained from patients or their parents or guardians. Ethical approval was provided by the joint The Gambian Government/MRC Joint Ethics Committee.

\section{Genotyping}

Polymorphisms in MSR1 and MARCO genes were identified from the National Center for Biotechnology Information dbSNP database (http://www.ncbi.nlm.nih.gov/projects/SNP/). SNPs were genotyped using the Sequenom (San Diego) MassARRAY system, and the primer extension products were analyzed using MALDI-TOF mass spectrometry [25,26].

\section{Bioinformatic analysis}

Potential alternative splice sites of MARCO were investigated using the Alternative Splice Site Predictor (ASSP) tool (http://wangcomputing.com/assp/index.html) [27]. Alignments of intron 1 and regions of high homology between multiple species were determined using ESPERR (evolutionary and sequence pattern extraction through reduced representations) [28]. To investigate whether there might be conserved transcriptional elements in intron 1 of the gene encoding MARCO, the Transcription Element Search System (TESS) program was used (http://www.cbil.upenn.edu/tess) [29].

\section{Statistical analysis}

Statistical analysis of genotype associations was performed using SPSS version 12.0 (SPSS, Inc., Chicago, IL). Analysis of linkage disequilibrium (LD) and haplotypes (Figure 1) was performed using the Haploview version 3.2 program [30]. Haplotype blocks were defined as regions demonstrating strong evidence of historical recombination between less than $5 \%$ of single-nucleotide polymorphism (SNP)-pair comparisons [31]. All control genotype distributions were in Hardy-Weinberg equilibrium (0.05 level).

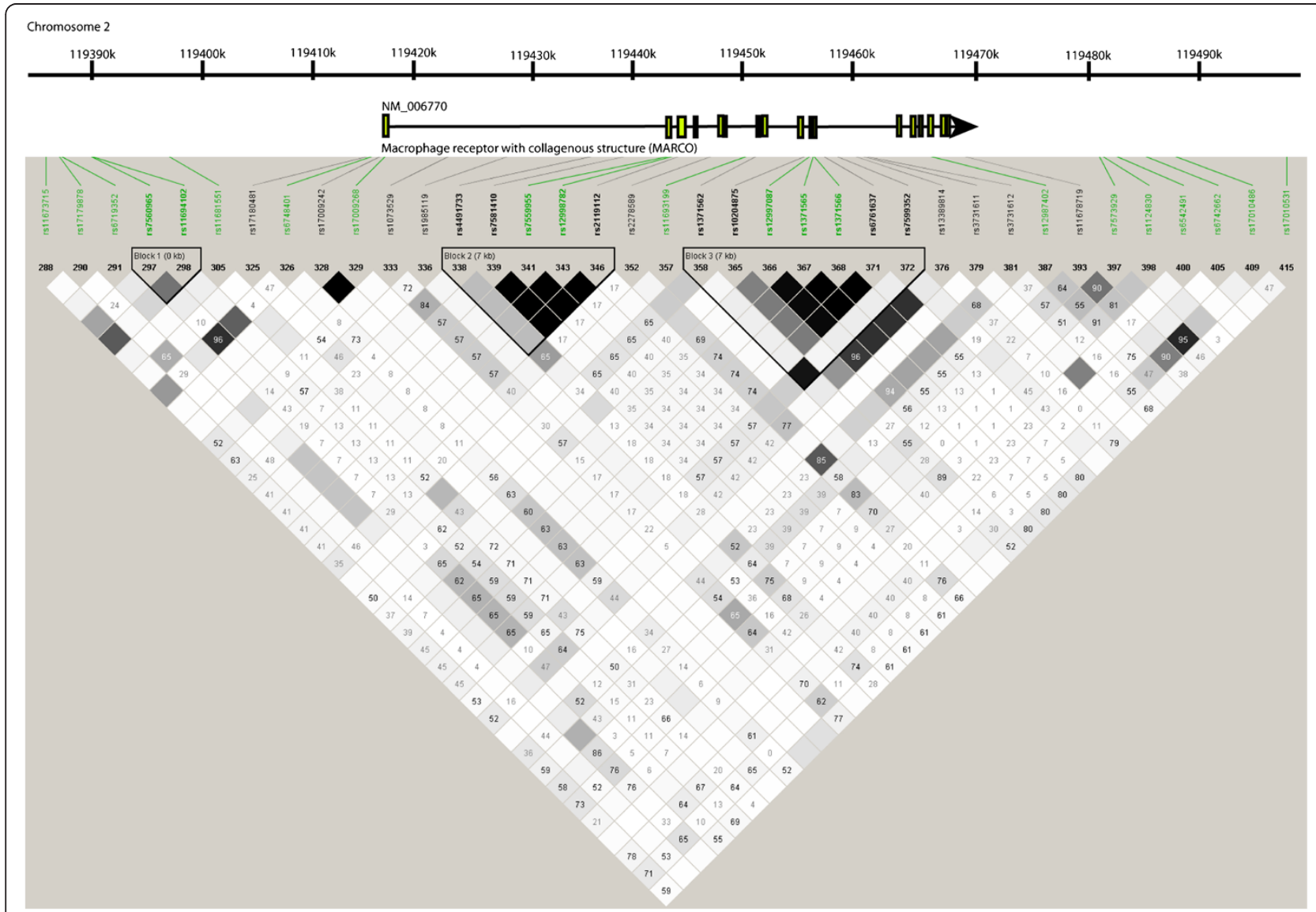

Figure 1 Haploview analysis of SNPs in MARCO [GeneID: 8685], located on chromosome 2q12. Linkage Disequilibrium (LD) in the African population is visualized across the MARCO locus, including upstream and downstream regions (chr2: 119414167-119468337). Polymorphisms are identified by their dbSNP rs numbers, and their position relative to the gene structure is marked. SNPs marked in bold were typed in this study. SNPs marked in green were included in the Wellcome Trust Case Control consortium [32] analysis and were used in validation studies. Empty squares indicate a high degree of $L D\left(L D\right.$ coefficient $\left.D^{\prime}=1\right)$ between pairs of markers. Numbers indicate the $D^{\prime}$ value expressed as a percentile. Black squares indicate pairs in strong LD with logarithm of odds (LOD) scores for $L D \geq 2$; grey squares, $D^{\prime}<1$ with LOD $\geq 2$; white squares, $D^{\prime}<1.0$ and $L O D<2$. 


\section{Results}

SNPs in MARCO are associated with resistance/ susceptibility to tuberculosis

We chose 25 tag SNPs in MARCO and 22 in MSR1; preliminary analysis indicated that these SNPs were polymorphic and found at reasonable frequencies in the Gambian population (e.g. > 5\%). None of the SNPs genotyped in MSR1 were associated with tuberculosis susceptibility (Additional file 1 Table S1). Three SNPs in MARCO showed some evidence of association with susceptibility to tuberculosis $(p<0.02$, Table 2), and for one of these, rs7559955, heterozygotes showed reduced risk of tuberculosis $(p=0.008$, Table 3$)$. These SNPs were located within two regions of the MARCO gene. The first cluster of three SNPs is located in intron 1 of the gene (rs4491733, rs7559955, rs12998782), and 1 SNP (rs13389814) was found in intron 16.

The T variant in rs7559955 may confer a regulatory element The presence of a polymorphism in rs7559955 is associated with resistance to tuberculosis and may confer

Table 2 Association analysis of MARCO SNPs in a Gambian tuberculosis case control study

\begin{tabular}{|c|c|c|c|c|c|c|}
\hline \multirow{2}{*}{$\frac{\text { CHR }}{2}$} & \multirow{2}{*}{$\begin{array}{l}\text { SNP } \\
\text { rs17180481 }\end{array}$} & \multirow{2}{*}{$\begin{array}{l}\text { Position } \\
119414167\end{array}$} & \multicolumn{2}{|c|}{ Genotypic } & \multicolumn{2}{|c|}{ Dominant } \\
\hline & & & 5.2 & 7.60E-02 & 4.2 & 4.10E-02 \\
\hline 2 & rs6748401 & 119414287 & 3.9 & 1.40E-01 & 0 & 8.30E-01 \\
\hline 2 & rs6748401 & 119414527 & 2.6 & 2.80E-01 & 0.5 & 4.90E-01 \\
\hline 2 & rs17009242 & 119415253 & 1.6 & 4.50E-01 & 1.6 & 2.10E-01 \\
\hline 2 & rs17009268 & 119415479 & NA & $\mathrm{NA}$ & NA & NA \\
\hline 2 & rs1073529 & 119421449 & 1.8 & 4.20E-01 & 1.7 & $1.90 \mathrm{E}-01$ \\
\hline 2 & rs1985119 & 119426870 & 1.3 & 5.20E-01 & 1.2 & 2.70E-01 \\
\hline 2 & rs4491733 & 119434498 & 12.3 & 2.10E-03 & 6.5 & 1.10E-02 \\
\hline 2 & rs7559955 & 119441199 & 7.8 & $2.00 \mathrm{E}-02$ & 6.9 & 8.50E-03 \\
\hline 2 & rs12998782 & 119441610 & 7.3 & $2.60 \mathrm{E}-02$ & 6.5 & 1.10E-02 \\
\hline 2 & rs2119112 & 119442389 & 1 & $6.10 \mathrm{E}-01$ & 0.4 & 5.10E-01 \\
\hline 2 & rs2278589 & 119445346 & 0 & 9.90E-01 & 0 & 9.60E-01 \\
\hline 2 & rs11693199 & 119448272 & 5.9 & $5.10 \mathrm{E}-02$ & 3.1 & 7.90E-02 \\
\hline 2 & rs1371562 & 119449418 & 0.1 & 9.30E-01 & 0.1 & 7.10E-01 \\
\hline 2 & rs10204875 & 119453881 & 2.9 & $2.40 \mathrm{E}-01$ & 1.4 & 2.30E-01 \\
\hline 2 & rs12997087 & 119454047 & 0.6 & 7.60E-01 & 0.4 & $5.20 \mathrm{E}-01$ \\
\hline 2 & rs1371565 & 119454355 & 0.5 & 7.80E-01 & 0.5 & 4.90E-01 \\
\hline 2 & rs1371566 & 119454417 & 0.5 & 7.60E-01 & 0.4 & 5.50E-01 \\
\hline 2 & rs6761637 & 119455533 & 1.1 & 5.80E-01 & 1 & $3.20 \mathrm{E}-01$ \\
\hline 2 & rs7599352 & 119456760 & 1.5 & 4.70E-01 & 1.1 & 2.90E-01 \\
\hline 2 & rs13389814 & 119458420 & 8 & $1.90 \mathrm{E}-02$ & 6.9 & 8.50E-03 \\
\hline 2 & rs3731611 & 119459081 & 1.7 & 4.30E-01 & 1.6 & 2.10E-01 \\
\hline 2 & rs3731612 & 119459335 & 1.7 & 4.30E-01 & 1.6 & 2.10E-01 \\
\hline 2 & rs12987402 & 119465026 & 1.6 & 4.40E-01 & 1.3 & 2.50E-01 \\
\hline 2 & rs11678719 & 119468337 & 5.1 & 7.70E-02 & 0.7 & 4.00E-01 \\
\hline
\end{tabular}

Table 3 The T allele of rs7559955 shows heterozygote protection

\begin{tabular}{lrrr}
\hline & Control & Tuberculosis & Total \\
\hline $\mathbf{C / C}$ & $\mathbf{5 4 2}$ & $\mathbf{5 8 1}$ & 1123 \\
$\mathbf{C} / \mathbf{T}$ & $\mathbf{6 5 1}$ & $\mathbf{5 5 3}$ & 1204 \\
$\mathbf{T} / \mathbf{T}$ & $\mathbf{1 5 6}$ & $\mathbf{1 5 0}$ & 306 \\
total & 1349 & 1284 & 2633 \\
& $40.2 \%$ & $45.2 \%$ & \\
& $48.3 \%$ & $43.1 \%$ & \\
& $11.6 \%$ & $11.7 \%$ & \\
HW P & 0.060 & 0.295 & \\
\hline
\end{tabular}

heterozygote protection (Table 3). Bioinformatic analysis using ESPERR indicates that the 200 bp region surrounding rs7559955 contains a relatively short sequence that is highly conserved amongst seven species (human, chimpanzee, macaque, mouse, rat, dog, and cow) and is thus a potential regulatory sequence (Figure 2A). Score values above the 0.1 default upper limit indicate very marked resemblance to alignment patterns typical of regulatory elements [33,34]. We thus hypothesized that this region might be a transcription factor binding site. Analysis using Transcription Element Search System (TESS) indicated that there was a weak match in both the ancestral and variant allele for the transcription factor NF-1 (score 8.0). The T allele appears to result in the addition of a transcription factor binding site that has homology to that of EFII (score 9.155) or C/EBPalpha (score 10) [29] (Figure 2B).

\section{Discussion}

Herein, we demonstrate that polymorphisms within the human class A scavenger receptor MARCO correlate with susceptibility/resistance to tuberculosis in a Gambian population. Interestingly, none of the SNPs identified in this study encoded non-synonymous mutations, but rather were within introns (i.e. rs13389814 within intron 16) and putative regulatory regions (i.e. rs7559955 within a potential promoter site in intron 1). This may imply that changes in MARCO expression rather than overt changes in structure are key to conferring resistance or susceptibility to tuberculosis. In fact, although SRA (encoded by MSR1) shares high amino acid similarity with MARCO (76\% amino acid similarity) and has many overlapping ligands, the two receptors differ considerably at the level of regulation of expression. Whereas SRA expression is regulated primarily by factors associated with macrophage differentiation (e.g. GM-CSF) and lipid accumulation, MARCO is primarily regulated by inflammation and bacterial infection (reviewed in [35]). Unlike SRA, which is expressed on virtually all macrophages, MARCO is constitutively expressed on some subsets of macrophages (i.e. alveolar, peritoneal, 


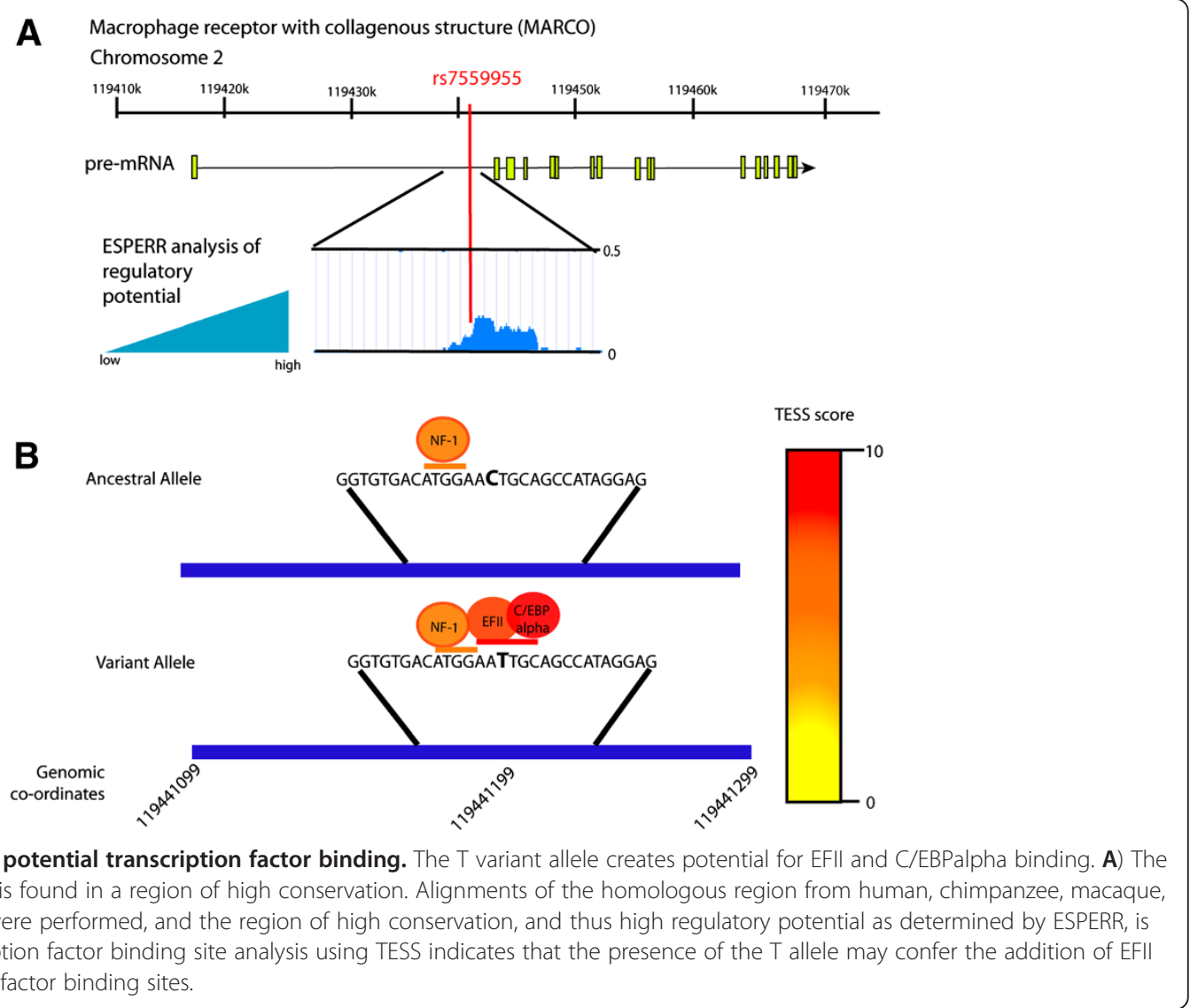

marginal zone of the spleen, and medullary cords of the lymph nodes), and is rapidly up-regulated on others during the course of infection. Indeed, in vivo MARCO expression is quickly $(<1 \mathrm{~h})$ increased upon challenge with bacteria or bacterial products, and this increase in expression occurs even at sites distal to bacterial challenge (e.g. on Kupffer cells post lung infection) [36,37]. Changes in MARCO expression during the course of experimental tuberculosis infections have not been performed, but expression of MARCO increases upon systemic challenge with BCG [37] and is higher on macrophages within and proximal to BCG-containing granulomas. MARCO-expressing macrophages in the marginal zone appear to phagocytose more BCG than neighbouring macrophages that do not express MARCO [9]. Whether the presence of a SNP within intron 1 (rs7559955) results in enhanced or decreased expression of MARCO, constitutively or after exposure to pathogens or their products, and whether changes in expression alter binding, uptake, or cytokine production, requires further investigation.

Recently Ma et al. reported that SNPs in MARCO were associated with susceptibility to tuberculosis in the Chinese Han population [23]. Interestingly, they identified that rs17009716 (chromosomal position 119441930), was significantly associated with susceptibility to tuberculosis.
This SNP is also within intron 1, less than 400 base pairs from and in high LD with rs12998782 and rs7559955 in Chinese (CHB), European (CEU), and African (YRI) populations. This provides support for potential consistency of a MARCO association across diverse populations and suggests a role for MARCO, in host defence against tuberculosis, possibly at the level of gene regulation, although further functional studies are warranted. Although animal models using MARCO knockout mice strongly associate MARCO with many pulmonary infections, such as pneumonia and influenza [38-41], whether polymorphisms in MARCO are associated with susceptibility to infection in humans remains to be determined. A recent Danish study found that non-synonymous SNPs within MARCO were found at very low frequency (0.005-5\%) within a Danish population, and in a study investigating whether MARCO was associated with chronic obstructive pulmonary disease (COPD) and lung infection in COPD patients, only one of these was associated with an increased risk of sepsis and none with pneumonia or COPD itself [42]. Unfortunately, the intronic SNPs identified in our study and in the Chinese Han population [23] were not investigated. It will be essential to validate these studies in more diverse populations, and perhaps to investigate more SNPs, in order to confirm the relevance of these results. 
The observed association of MARCO variants with sepsis is consistent with in vivo $[36,37,43,44]$ and in vitro $[44,45]$ observations that MARCO regulates pro-inflammatory cytokine production to whole bacteria and TLR ligands. The class A scavenger receptors in general, and MARCO in particular, have been demonstrated to enhance TLR signalling to mycobacterial cell wall components, such as the major immunogenic lipid on the mycobacterial cell wall, trehalose dimycolate (TDM) [46]. Whether this increased regulation of cytokines is of benefit or is detrimental to the host during the course of either pulmonary or systemic tuberculosis infection remains to be determined.

\section{Conclusions}

In summary, we have demonstrated that genetic variation in a class A scavenger receptor, MARCO, is associated with susceptibility/resistance to tuberculosis in a Gambian population, consistent with previously reported data from a Chinese Han population [23]. Further studies are warranted to determine whether genetic variation in MARCO may alter expression of the receptor, and whether regulation of MARCO expression contributes to protection or susceptibility to tuberculosis at the level of macrophage infection, initiation of a pro-inflammatory response, or long-term persistence.

\section{Additional file}

Additional file 1: Table S1. Genotyped SNPS in MSR1.

\section{Abbreviations}

SR: Scavenger receptor; SNP: Single nucleotide polymorphism; TLR: Toll-like receptor; MARCO: Macrophage receptor with collagenous structure; WTCCC: Wellcome Trust Case Control Consortium; SRCR: Scavenger receptor cysteine-rich domain; PBMC: Peripheral blood mononuclear cell.

\section{Competing interests}

The authors have no competing interests.

\section{Authors' contributions}

DMEB, KS, NL and FOV performed experiments. PCH, GS, MN, AVSH provided infrastructure and support for sample collection and processing. DMEB, SG, AVSH, FOV designed the experiments. FOV performed the statistical analysis. KS, SG, and AVSH provided funding. All authors read, edited, commented and approved on the manuscript.

\section{Acknowledgements}

This work was funded by the Wellcome Trust. A.V.S.H. was supported by a Wellcome Trust Principal Fellowship. This work was funded in part by $\mathrm{NIH}$ Grant Number 1 R15 Al094436-01A1 to KS. DMEB would like to thank Dr. Chris Verschoor for critical reading of the manuscript. Work in the Bowdish laboratory is supported in part by the McMaster Immunology Research Centre and the M. G. DeGroote Institute for Infectious Disease Research.

\section{Author details}

${ }^{1}$ McMaster Immunology Research Centre, McMaster University, Hamilton, ON L8S 4K1, Canada. 'Department of Pathology, College of Veterinary Medicine, University of Georgia, 501 D.W. Brooks Drive, Athens, GA 30605, USA. ${ }^{3}$ School of Medicine, Koc University, Istanbul 34450, Turkey. ${ }^{4}$ Medical Research Council Laboratories, Fajara, The Gambia. ${ }^{5}$ Centre for International Health, Department of Preventive and Social Medicine, University of Otago, School of Medicine, Dunedin, New Zealand. ${ }^{6}$ Ospedale San Pietro Fatebenefratelli, Rome, Italy. ${ }^{7}$ Medical School Research Building, Brighton \& Sussex Medical School, Brighton, East Sussex BN1 9PS, UK. ${ }^{8}$ Sir William Dunn School of Pathology, University of Oxford, 10 South Parks Road, Oxford OX1 3RE, UK. 9The Wellcome Trust Centre for Human Genetics, Roosevelt Drive, Oxford OX3 7BN, UK. ${ }^{10}$ School of Biology, Georgia Institute of Technology, 310 Ferst Drive, Atlanta, GA 30332, USA.

Received: 19 December 2012 Accepted: 16 April 2013

Published: 23 April 2013

\section{References}

1. Organization, W.H. Global tuberculosis control: WHO report 2011. ; 2011. cited 2011; Available from: http://apps.who.int/iris/bitstream/10665/75938/1/ 9789241564502_eng.pdf.

2. Schlesinger LS: Macrophage phagocytosis of virulent but not attenuated strains of mycobacterium tuberculosis is mediated by mannose receptors in addition to complement receptors. J Immunol 1993, 150(7):2920-2930.

3. Maeda N, et al: The cell surface receptor DC-SIGN discriminates between mycobacterium species through selective recognition of the mannose caps on lipoarabinomannan. J Biol Chem 2003, 278(8):5513-5516.

4. Schlesinger LS, et al: Phagocytosis of mycobacterium tuberculosis is mediated by human Monocyte complement receptors and complement component C3. J Immunol 1990, 144(7):2771-2780.

5. Rothfuchs $A G$, et al: Dectin-1 interaction with mycobacterium tuberculosis leads to enhanced IL-12p40 production by splenic dendritic cells. J Immunol 2007, 179(6):3463-3471.

6. Montoya D, et al: Divergence of macrophage phagocytic and antimicrobial programs in leprosy. Cell Host Microbe 2009, 6(4):343-353.

7. Bermudez LE, Parker A, Goodman JR: Growth within macrophages increases the efficiency of mycobacterium avium in invading other macrophages by a complement receptor-independent pathway. Infect Immun 1997, 65(5):1916-1925.

8. Haworth $\mathrm{R}$, et al: The macrophage scavenger receptor type $\mathrm{a}$ is expressed by activated macrophages and protects the host against lethal endotoxic shock. J Exp Med 1997, 186(9):1431-1439.

9. Ito $S$, et al: Roles of a macrophage receptor with collagenous structure (MARCO) in host defense and heterogeneity of splenic marginal zone macrophages. Arch Histol Cytol 1999, 62(1):83-95.

10. Zimmerli S, Edwards S, Ernst JD: Selective receptor blockade during phagocytosis does not alter the survival and growth of mycobacterium tuberculosis in human macrophages. Am J Respir Cell Mol Biol 1996, 15(6):760-770

11. Pedroza-Gonzalez A, et al: In situ analysis of lung antigen-presenting cells during murine pulmonary infection with virulent mycobacterium tuberculosis. Int J Exp Pathol 2004, 85(3):135-145.

12. Court $\mathrm{N}$, et al: Partial redundancy of the pattern recognition receptors, scavenger receptors, and C-type lectins for the long-term control of mycobacterium tuberculosis infection. J Immunol 2010, 184(12):p 7057-p 7070.

13. Neyrolles $\mathrm{O}$, et al: Is adipose tissue a place for mycobacterium tuberculosis persistence? PLoS One 2006, 1:e43.

14. Jozefowski S, et al: Mycobacterium tuberculosis lipoarabinomannan enhances LPS-induced TNF-alpha production and inhibits NO secretion by engaging scavenger receptors. Microb Pathog 2011, 50(6):p.350-p.359.

15. Drage MG, et al: TLR2 And its co-receptors determine responses of macrophages and dendritic cells to lipoproteins of mycobacterium tuberculosis. Cell Immunol 2009, 258(1):29-37.

16. Schoenen $\mathrm{H}$, et al: Cutting edge: mincle is essential for recognition and adjuvanticity of the mycobacterial cord factor and its synthetic analog trehalose-dibehenate. J Immunol 2010, 184(6):p 2756-p 2760.

17. Bowdish DM, et al: MARCO, TLR2, and CD14 are required for macrophage cytokine responses to mycobacterial trehalose dimycolate and mycobacterium tuberculosis. PLoS Pathog 2009, 5(6):e1000474.

18. Sever-Chroneos Z, et al: Prolonged survival of scavenger receptor class Adeficient mice from pulmonary mycobacterium tuberculosis infection. Tuberculosis (Edinb) 2011, 91 Suppl 1:p. S69-p. S74. 
19. Suzuki $H$, et al: A role for macrophage scavenger receptors in atherosclerosis and susceptibility to infection. Nature 1997, 386(6622):292

20. Apt A, Kramnik I: Man and mouse TB: contradictions and solutions. Tuberculosis (Edinb) 2009, 89(3):195-198.

21. Bennett $S$, et al: Investigation of environmental and host-related risk factors for tuberculosis in Africa II. Investigation of host genetic factors. Am J Epidemiol 2002, 155(11):1074-1079.

22. Lienhardt C, et al: Investigation of environmental and host-related risk factors for tuberculosis in africa I. Methodological aspects of a combined design. Am J Epidemiol 2002, 155(11):1066-1073.

23. Ma MJ, et al: Genetic variants in MARCO are associated with the susceptibility to pulmonary tuberculosis in Chinese Han population. PLoS One 2011, 6((8):p. e24069.

24. Jallow $\mathrm{M}$, et al: Genome-wide and fine-resolution association analysis of malaria in west Africa. Nat Genet 2009, 41(6):657-665.

25. Jurinke $C$, et al: The use of MassARRAY technology for high throughput genotyping. Adv Biochem Eng Biotechnol 2002, 77:57-74.

26. Jurinke $C$, et al: Automated genotyping using the DNA MassArray technology. Methods Mol Biol 2002, 187:179-192.

27. Wang M, Marin A: Characterization and prediction of alternative splice sites. Gene 2006, 366(2):219-227.

28. Taylor J, et al: ESPERR: learning strong and weak signals in genomic sequence alignments to identify functional elements. Genome Res 2006 16(12):1596-1604.

29. Schug J: Using TESS to predict transcription factor binding sites in DNA sequence. Curr Protoc Bioinformatics 2008, Chapter 2:p. Unit 2-p. Unit 6.

30. Barrett JC, et al: Haploview: analysis and visualization of LD and haplotype maps. Bioinformatics 2005, 21(2):263-265.

31. Gabriel SB, et al: The structure of haplotype blocks in the human genome. Science 2002, 296(5576):2225-2229.

32. Thye T, et al: Genome-wide association analyses identifies a susceptibility locus for tuberculosis on chromosome 18q11.2. Nat Genet 2010, 42(9):p 739-p 741

33. King DC, et al: Evaluation of regulatory potential and conservation scores for detecting cis-regulatory modules in aligned mammalian genome sequences. Genome Res 2005, 15(8):1051-1060.

34. Kolbe $D$, et al: Regulatory potential scores from genome-wide three-way alignments of human, mouse, and rat. Genome Res 2004, 14(4):700-707

35. Bowdish DM, Gordon S: Conserved domains of the class a scavenger receptors: evolution and function. Immunol Rev 2009, 227(1):19-31.

36. van der Laan $L$, et al: Macrophage scavenger receptor MARCO: in vitro and in vivo regulation and involvement in the anti-bacterial host defense. Immunol Lett 1997, 57(1-3):203-208.

37. van der Laan $L$, et al: Regulation and functional involvement of macrophage scavenger receptor MARCO in clearance of bacteria in vivo. J Immunol 1999, 162(2):939-947.

38. Arredouani $M$, et al: The scavenger receptor MARCO is required for lung defense against pneumococcal pneumonia and inhaled particles. J Exp Med 2004, 200(2):267-272.

39. Arredouani MS, et al: The macrophage scavenger receptor SR-AI/II and lung defense against pneumococci and particles. Am J Respir Cell Mol Biol 2006, 35(4):474-478.

40. Palecanda A, Kobzik L: Receptors for unopsonized particles: the role of alveolar macrophage scavenger receptors. Curr Mol Med 2001, 1(5):589-595.

41. Ghosh S, et al: MARCO regulates early inflammatory responses against influenza: a useful macrophage function with adverse outcome. Am J Respir Cell Mol Biol 2011, 45(5):p 1036-p 1044

42. Thomsen M, et al: Genetic variation in the scavenger receptor MARCO and its association with chronic obstructive pulmonary disease and lung infection in 10,604 individuals. Respiration.

43. Elomaa O, et al: Structure of the human macrophage MARCO receptor and characterization of its bacteria-binding region. J Biol Chem 1998 273(8):4530-8

44. Dorrington M, Roche A, Chauvin SE, Tu Z, Mossman K, Weiser JN, Bowdish DME: MARCO is required for TLR2- and NOD2-mediated responses to streptococcus pneumoniae and clearance of pneumococcal colonization in the murine nasopharynx. J Immunol 2013, 190(1):p 250-8.
45. Jozefowski $S$, et al: Disparate regulation and function of the class a scavenger receptors SR-AI/II and MARCO. J Immunol 2005, 175(12):8032-41

46. Bowdish DME, Sakamoto K, Mukhopadhyay S, Leifer CA, Tryvaggson K Gordon S, Russell DG: MARCO is a binding receptor for mycobacterial trehalose 6,6'-dimycolate and is required to initiate TLR2 induced signaling. In preparation 2008.

doi:10.1186/1471-2350-14-47

Cite this article as: Bowdish et al: Genetic variants of MARCO are associated with susceptibility to pulmonary tuberculosis in a Gambian population. BMC Medical Genetics 2013 14:47.

\section{Submit your next manuscript to BioMed Central and take full advantage of:}

- Convenient online submission

- Thorough peer review

- No space constraints or color figure charges

- Immediate publication on acceptance

- Inclusion in PubMed, CAS, Scopus and Google Scholar

- Research which is freely available for redistribution

Submit your manuscript at www.biomedcentral.com/submit
C) Biomed Central 